\section{Avaliação da Indissociabilidade entre Ensino, Pesquisa e Extensão Relacionada à Promoção do Desenvolvimento Infantil em Instituições de Ensino Superior do Interior Paulista}

\author{
Evaluation of Inextricableness among Teaching, Research \\ and Extension on Child Development in High Education \\ Institutions at Municipalities in São Paulo, Brazil
}

\title{
RESUMO
}

Este artigo propõe um esquema analítico para a avaliação da incorporação de conteúdos relacionados ao desenvolvimento infantil (DI) à luz da indissociabilidade entre ensino, pesquisa e extensão. Analisou-se um programa desenvolvido por uma fundação do terceiro setor que articulou representantes da comunidade local, das instituições de ensino superior (IES) e dos setores da saúde, educação e assistência social de cinco municípios no interior paulista. Evidências da neurociência, epigenética e sociologia da infância indicam a importância dos investimentos sociais na primeira infância (PI). Tem-se como objetivo conhecer a inclusão de conteúdos inovadores de DI nos âmbitos de ensino, pesquisa e extensão dessas IES partícipes. Realizou-se uma pesquisa-ação com 15 coordenadores de 8 cursos de graduação que responderam a um questionário e forneceram as ementas e planos de ensino de 47 disciplinas e atividades de pesquisa e de extensão. Os cursos avaliados incluíam o ensino dos conteúdos de DI sem incorporar as evidências recentes e, tampouco, articulavam a pesquisa e a extensão. Tópicos essenciais para o cuidado integral e integrado na PI são pouco abordados na maioria dos cursos. Considera-se que o esquema analítico proposto evidenciou como ocorre a articulação ensino-pesquisa-extensão relacionada à PI nos cursos avaliados, contribuindo para o alcance da integração desejada.

Palavras-chave: Extensão Universitária. Relações Comunidade-Instituição. Avaliação de Programas e Projetos de Saúde. Instituições de Ensino Superior. Primeira Infância.

\section{ABSTRACT}

This paper proposes an analysis scheme to evaluate incorporation of contents related to child development (CD) in light of inextricableness among teaching, research and extension in Higher Education Institutions (HEI). We have analyzed a program created by a Third Sector Foundation which articulated community members, HEI
Alfredo Almeida

Pina-Oliveira, AnNa

Maria Chiesa, Roseli

DE LANA MOREIRA E

Rosemary Aparecida

Fracolli Pécora

Universidade de São Paulo. Escola de Enfermagem, São Paulo, Brasil 
representatives and workers from Health, Education and Social Welfare at five municipalities in country area of Sao Paulo, Brazil. Strong evidences from Neuroscience, Epigenetics, and Sociology indicate the need to widen social investments on early childhood. We aim to know how HEI present innovative contents on CD into teaching, research and extension. We have conducted an action-research with 15 coordinators of 8 undergraduate courses who filled a questionnaire and provided summaries and syllabi from 47 disciplines and research/extension activities. Most of evaluated courses handle CD teaching without updated evidences, neither makes research nor extension. Essential topics related to foster an integral and integrated early childhood care were slightly approached on them. The proposed analysis scheme has contributed to substantiate how teaching-research-extension inextricableness related to $\mathrm{CD}$ occurs and has improved body of knowledge on this field.

Keywords: Extension. Community-Institutional Relations. Program Evaluation. Higher Education Institutions. Child Development.

\section{INTRODUÇÃO}

A qualificação da força de trabalho, desde a graduação universitária, consiste em um grande desafio do século XXI em vários países, sobretudo relacionado aos profissionais da área da saúde. Processos educativos devem possibilitar a inserção dos estudantes nos serviços a fim de que estes aprendam com situações concretas de trabalho e atuem com maior resolubilidade e efetividade a partir de experiências educativas baseadas em competências globais e locais para o cuidado em saúde [9].

Nesse cenário em construção, as atividades de extensão representam oportunidades para articular o ensino e a pesquisa a fim de promover boas práticas profissionais, participação comunitária, engajamento social e mudanças na realidade na qual se inserem com possibilidades para construir uma formação democrática e emancipatória que se contraponha à globalização neoliberal e à mercantilização do conhecimento $[17,20]$.

Construir indicadores da extensão pode influenciar na organização da gestão do ensino superior a fim de superar lacunas e celebrar conquistas relacionadas à avaliação sistemática da tríade acadêmica que compreende o ensino, a pesquisa e a extensão de modo indissociável e que legitima o compromisso social das diferentes instituições de ensino superior (IES) com a comunidade em que se inserem $[3,6]$.

Nesse sentido, o presente estudo pretende contribuir para a elaboração de formas de avaliar a indissociabilidade entre ensino, pesquisa e extensão a partir de uma experiência relacionada à transferência de tecnologias e incorporação de evidências para a promoção do desenvolvimento infantil (DI) derivada de uma parceria entre a Fundação Maria Cecília Souto Vidigal* (FMCSV) e cinco municípios do interior paulista.

\footnotetext{
*Entende-se que o conceito de primeira infância compreende a vida intrauterina até os seis primeiros anos de vida. Entretanto, a referida fundação tem atuado na produção, tradução e disseminação de conhecimentos relacionados às políticas e práticas que fortalecem a "primeiríssima infância" com ênfase nos três primeiros anos de vida. Disponível em: www.fmcsv.org.br. Acesso em: 23 mar. 2014.
} 
Cada município firmou um acordo para a implementação de ações do Programa de Desenvolvimento Infantil (PDI) da FMCSV com o intuito de aperfeiçoar a aplicação de recursos públicos e privados e fortalecer as competências de diferentes stakeholders $^{* *}$ que atuam, direta ou indiretamente, nos cuidados desde a vida intrauterina até os três anos de vida, fase denominada como "primeiríssima infância" (PI). Para tanto, solicitou-se o compromisso de prefeitos, representantes dos setores da saúde, educação e assistência social, membros da comunidade local e responsáveis de instituições de ensino superior para a consolidação dos projetos de intervenção local (PIL) [10].

O objetivo do PDI da FMCSV consiste em promover e intensificar os cuidados qualificados em prol dessa fase da vida com base nas evidências científicas advindas da neurociência e da epigenética, reconhecidas por Cypel [7] como conteúdos inovadores para "a organização dos alicerces que favorecerão o indivíduo, no futuro, a lidar com as complexas situações que a vida venha a lhe apresentar”. Não obstante, o autor ainda identifica a contradição dos programas dedicados à PI que atuam de forma fragmentada, ora na saúde, ora na educação, ora nos demais setores $[7,10]$.

Com a finalidade de superar tal fragmentação do conhecimento e das práticas, a fundação elaborou os PIL a fim de proporcionar as bases para se adotar um cuidado integral - que extrapola a dimensão biológica e valoriza aspectos emocionais e socioambientais - e integrado - que articula diferentes setores e áreas do conhecimento da criança de zero a três anos, com a inclusão de suas famílias e sua comunidade [10].

Esses projetos de intervenção são compostos por conteúdos inovadores que traduzem a síntese de evidências da PI que demonstram efeitos positivos: indicam a promoção da saúde, da autoestima, da segurança e do sucesso profissional dos indivíduos, enquanto que, no âmbito das famílias e das comunidades, tal rede de cuidados contribui para o desenvolvimento social com equidade e para a redução do consumo de drogas, da criminalidade e da violência $[4,7,12,18,20]$.

Tais conteúdos inovadores foram disseminados por meio de oito intervenções-chave (IC), que se organizaram em dois momentos [10]. O primeiro foi composto por oficinas de formação com profissionais dos setores da saúde, educação e assistência social, bem como outros profissionais envolvidos com a PI no município. $\mathrm{O}$ segundo foi dividido em três supervisões periódicas (bimestrais ou trimestrais) a fim de verificar a aplicabilidade e a replicação dos conteúdos do desenvolvimento infantil (DI) com as equipes nos serviços de origem. Os representantes das IES - estudantes de graduação e de pós-graduação, docentes, coordenadores e diretores - também poderiam participar das IC.

Ao longo desse processo, os consultores das oito intervenções da FMCSV criaram oportunidades para a incorporação da síntese das evidências das ciências biomédicas e ciências da saúde - concorrendo para a promoção do DI - aliadas ao corpo de conhecimento das ciências humanas e ciências sociais a fim de promover, de modo também

\footnotetext{
**Stakeholder significa público estratégico, parte interessada ou interveniente. Termo em inglês muito utilizado nas áreas de comunicação, administração e tecnologia da informação cujo objetivo é designar as pessoas e grupos mais importantes para um planejamento estratégico ou plano de negócios, ou seja, as partes interessadas.
} 
indissociável, o cuidar, o brincar e o educar na PI $[4,7,10,20]$.

A proposição do esquema para análise da tríade acadêmica foi fundamentada na lacuna de indicadores de extensão que demonstram como as IES incorporam tais avanços científicos na graduação de diferentes cursos. Nesse contexto, o presente estudo objetivou conhecer a inclusão dos conteúdos inovadores de desenvolvimento infantil nos âmbitos de ensino, pesquisa e extensão dessas instituições de ensino superior partícipes, antes da implementação dos projetos de intervenção local da Fundação Maria Cecília Souto Vidigal.

\section{MATERIAL E MÉTODO}

Trata-se de uma pesquisa-ação***, dado o caráter participativo, democrático e com potencial para a mudança social durante a produção do conhecimento sobre formas de avaliar a extensão, sem desconsiderar o ensino e a pesquisa no processo de incorporação de conteúdos relacionados ao DI fundamentados em evidências científicas a fim de promover um cuidado integral e integrado em prol da PI $[13,18,20]$.

Os sujeitos da pesquisa foram 15 coordenadores de cursos de graduação de um centro universitário comunitário (CUC), um centro universitário privado (CUP), uma fundação educacional particular (FEP), uma universidade com fins lucrativos (UFL) e uma universidade pública federal (UPF).

Os cenários do estudo foram organizados a partir dos cursos analisados com seus respectivos números de disciplinas apresentados em parênteses e dos dados sociodemográficos dos municípios que aderiram à parceria com a FMCSV (Tabela 1). Destaca-se que quatro dessas IES estão situadas no mesmo município que realizou tal acordo com a FMCSV e apenas um curso apresentou currículo integrado no ensino de graduação.

\footnotetext{
***Projeto decorrente da pesquisa composta por seis fases e intitulada A Universidade e a transferência de tecnologias de desenvolvimento infantil em municípios paulistas. Os resultados aqui apresentados são baseados na fase I e na discussão metodológica do esquema analítico desenvolvido pelos autores no grupo de pesquisa Modelos tecnoassistenciais e a Promoção da Saúde, liderado pela Profa. Dra. Anna Maria Chiesa, da Escola de Enfermagem da Universidade de São Paulo (EE-USP).
} 
Tabela 1 - Características das instituições de ensino superior (IES) e dados sociodemográficos dos municípios partícipes dos projetos de intervenção local (PIL) da Fundação Maria Cecília Souto Vidigal (FMCSV). São Paulo, 2014.

\begin{tabular}{|c|c|c|c|c|c|}
\hline \multirow{2}{*}{$\begin{array}{l}\text { DENOMINAÇÃO } \\
\text { DAS INSTITUIÇÕES } \\
\text { DE ENSINO } \\
\text { SUPERIOR (IES) } \\
\text { PARTÍCIPES**** }\end{array}$} & \multirow{2}{*}{$\begin{array}{l}\text { CURSOS E NÚMERO } \\
\text { DE DISCIPLINAS } \\
\text { QUE ABORDAM } \\
\text { DESENVOLVIMENTO } \\
\text { INFANTIL (DI) }\end{array}$} & \multicolumn{4}{|c|}{ DADOS SOCIODEMOGRÁFICOS DOS MUNICÍPIOS***** } \\
\hline & & $\begin{array}{l}\text { POPULAÇÃO } \\
\text { TOTAL }\end{array}$ & $\begin{array}{l}\text { ESPERANÇA } \\
\text { DE VIDA } \\
\text { AO NASCER } \\
\text { (ANOS) }\end{array}$ & $\begin{array}{l}\text { ÍNDICE DE } \\
\text { EXCLUSÃO } \\
\text { SOCIAL }\end{array}$ & $\begin{array}{l}\text { ÍNDICE DE } \\
\text { DESENVOLVIMENTO } \\
\text { HUMANO (IDH) }\end{array}$ \\
\hline $\begin{array}{l}\text { UNIVERSIDADE } \\
\text { PÚBLICA FEDERAL } \\
\text { (UPF) }\end{array}$ & $\begin{array}{l}\text { Fisioterapia (2) e Terapia } \\
\text { Ocupacional (3 módulos } \\
\text { de currículo integrado) }\end{array}$ & 226.322 & 73,08 & 0,55 & 0,841 \\
\hline $\begin{array}{l}\text { FUNDAÇÃO } \\
\text { EDUCACIONAL } \\
\text { PRIVADA (FEP) }\end{array}$ & $\begin{array}{l}\text { Pedagogia (2) e } \\
\text { Psicologia (5) }\end{array}$ & 59.096 & 71,65 & 0,59 & 0,810 \\
\hline $\begin{array}{l}\text { CENTRO } \\
\text { UNIVERSITÁRIO } \\
\text { COMUNITÁRIO } \\
\text { (CUC) }\end{array}$ & $\begin{array}{l}\text { Educação Física } \\
\text { (4), Enfermagem } \\
\text { (3), Fisioterapia (2), } \\
\text { Pedagogia (3) e } \\
\text { Psicologia (3) }\end{array}$ & 86.059 & 71,92 & 0,61 & 0,817 \\
\hline $\begin{array}{l}\text { CENTRO } \\
\text { UNIVERSITÁRIO } \\
\text { PRIVADO (CUP) }\end{array}$ & $\begin{array}{l}\text { Pedagogia (4) e } \\
\text { Psicologia (5) }\end{array}$ & 47.682 & 71,81 & 0,59 & 0,807 \\
\hline $\begin{array}{l}\text { UNIVERSIDADE } \\
\text { COM FINS } \\
\text { LUCRATIVOS (UFL) }\end{array}$ & $\begin{array}{l}\text { Enfermagem (5), } \\
\text { Fisioterapia (2), } \\
\text { Pedagogia (3) e } \\
\text { Psicologia (1) }\end{array}$ & 52.176 & 74,10 & 0,59 & 0,815 \\
\hline
\end{tabular}

Fonte: Divisão de Temas Educacionais do Itamaraty, 2014; Instituto Brasileiro de Geografia e Estatística, 2010.

Os coordenadores responderam a um questionário que contemplava a caracterização do curso, nove questões relacionadas ao ensino de DI, cinco sobre pesquisa e cinco com foco na extensão até o ano de 2009 (que antecedeu a parceria firmada em 2010 pela FMCSV entre os stakeholders municipais e as IES).

As ementas e planos de ensino dos oito cursos totalizaram 47 disciplinas que abordavam o cuidado de gestantes e de crianças até três anos com foco na família e na comunidade. A análise documental incidiu sobre essas disciplinas a fim de comparar e identificar

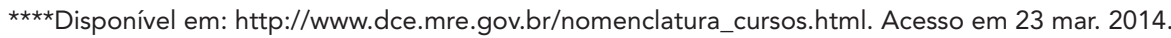

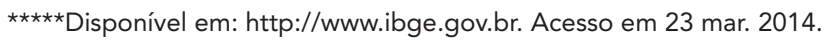


a presença dos temas centrais e tópicos do conteúdo programático das oito intervenções-chave (IC) da Fundação Maria Cecília Souto Vidigal (FMCSV) (Tabela 2).

Tabela 2 - Temas centrais das evidências científicas que fundamentam as IC da FMCSV e que orientaram a análise das ementas e planos de ensino das IES partícipes. São Paulo, 2014.

IC1. PRÉ-NATAL AMPLIADO

" Maternidade/Paternidade ou parentalidade

" Construção de vínculos familiares

" Aspectos emocionais da aceitação da gravidez

" Conflitos familiares

" Direitos da gestante

" Adesão às recomendações de pré-natal

" Indicadores de gravidade na gestação

"Presença de acompanhante no pré-natal e no parto

" Ultrassonografia como oportunidade de acolhimento

IC2. GRUPOS DE FAMIILIAS GRÁVIDAS
"Sentidos da maternidade e da família

" Medidas preventivas e educacionais

"Pré-natal para preservar o bem-estar físico e psíquico da gestante

" Garantia do acompanhamento mínimo do pré-natal

" Realização de exames pré-natais

Mudanças corporais da gestante

Mudanças emocionais da gestante

"Continência emocional da gestante e seu marido/ companheiro

" Rastreamento de transtornos emocionais do puerpério

IC3. PARTO HUMANIZADO

"Controle dos indicadores de saúde materna

" Garantia da equidade do acesso à maturidade

" Respeito à intimidade e às emoções do parto e nascimento

" Temores sobre prováveis vias do parto

" Medo de morrer no parto

" Preferências sobre o tipo de parto (normal, natural, cesárea)

" Medidas de redução da ansiedade do parto

" Desejo e/ou dúvidas sobre a capacidade de cuidar da criança

"Valorização do parto normal e natural

" Práticas não invasivas para o parto 
IC4. PUERPÉRIO E ALEITAMENTO MATERNO
" Adaptação da criança na família

"Adaptação da família à criança

" Dificuldades sentidas pela mãe em relação à adaptação com o novo bebê

" Importância da sistematização da visita domiciliária

" Demandas biológicas da mãe e do bebê

" Organização do cuidado no contexto familiar

"Valorização das competências familiares

"Incentivo ao aleitamento materno exclusivo e seus benefícios (físicos, afetivos e sociais)

" Identificação de medos e problemas emocionais da mãe no cuidado com o bebê

" Identificação das dificuldades relacionadas ao aleitamento materno exclusivo
IC5. PUERICULTURA AMPLIADA (ZERO A TRÊS ANOS)
"Políticas e recomendações nacionais sobre DI

"Políticas e recomendações internacionais sobre DI

"Benefícios imediatos do DI

"Benefícios futuros do DI

" Identificar as oportunidades de promoção do DI no contexto familiar, nas consultas com profissionais de saúde e sala de vacinação

" Avaliação da estimulação do bebê nos cuidados familiares

" Identificar medidas de segurança, apoio, afeto e amor ao bebê pela mãe (ou cuidador)

" Identificar medidas de alívio do incômodo e da dor frente a procedimentos invasivos

"Valorização da presença da mãe (ou cuidador) em momentos "estressantes" para o bebê em serviços de saúde
IC6. EDUCAÇÃO INFANTIL EM CRECHES
" Brincar como expressão corporal (movimento, gestos)

" Brincar como expressão artística (música, expressão plástica)

" Brincar como expressão escrita (desenho)

" Brincar como comunicação e expressão (emoções, medos, temperamento, criatividade, valores e atitudes)

" Garantia e respeito da participação da criança como sujeito de direitos e deveres

"Fortalecimento da autonomia da criança

"Valorização das necessidades e interesses da criança

"Estímulo ao desenvolvimento dos potenciais latentes nas crianças 
" Criação de espaços lúdicos comunitários

"Brincar como direito e exercício de cidadania

"Valorização do brincar no desenvolvimento integral das crianças de seis meses a três anos

" Espaço lúdico como promotor da convivência e socialização

" Respeito às diferenças individuais e diversidade cultural

" Espaço potencial de convivência familiar e participação comunitária

" Facilitar o acesso ao brinquedo/brincadeiras para crianças em diferentes situações sociais

" Estímulo ao lazer, cultura e cidadania da criança e sua família

"Adequação das atividades lúdicas em relação ao DI

IC8. GRUPOS DE FAMIILIAS COM CRIANÇAS DE ZERO A TRÊS ANOS DE IDADE
" Comunicação dos pais com a criança desde a vida intrauterina até os três anos

" Organização física do ambiente familiar para favorecer a sobrevivência física e psíquica da criança

"Valorização dos núcleos interpessoais na família

" Reconhecimento do DI nos seus aspectos físicos, cognitivos, emocionais, sociais e ambientais

"Vínculo e relação afetiva dos pais com a criança em relação ao crescimento saudável da criança

"Relações afetivas dos pais com os filhos como modelo de relacionamentos posteriores na vida

" Ênfase na tolerância, amor e acolhimento na construção da subjetividade da criança

" Comunicação dos pais com a criança desde a vida intrauterina até os três anos

" Organização física do ambiente familiar para favorecer a sobrevivência física e psíquica da criança

A partir do recorte da vida intrauterina até os três anos, os conteúdos inovadores relacionados ao DI descritos acima foram comparados com as respostas dos questionários dos coordenadores de curso e com as ementas e planos de aula de graduação adotados em 2009, permitindo a triangulação dos dados coletados a fim de fortalecer a caracterização da indissociabilidade da tríade acadêmica nas IES partícipes [14].

Em seguida, os autores desse estudo se reuniram a fim de identificar a presença dos tópicos das IC no material empírico supracitado e aplicar o esquema analítico, com destaque para a extensão, sem desconsiderar o ensino e a pesquisa, a saber:

" Ausência: os conteúdos das disciplinas das IES partícipes não abordam nenhuma das evidências científicas, sintetizados nos conteúdos do DI abordados nas IC da FMCSV, com atribuição de valor mínimo 1.

" Incipiente: relativa à presença dos tópicos das intervenções-chave (IC) exclusivamente no âmbito do ensino, dada a função formadora das IES, valor 2.

" Regular: relativa à presença dos tópicos das IC no ensino e pesquisa, sem considerar a extensão, valor 3 . 
") Relevante: relativa à presença dos tópicos das IC em dois âmbitos, sendo um destes a extensão, valor 4 .

" Completa: relativa à presença articulada que envolve a tríade ensino, pesquisa $\mathrm{e}$ extensão no processo de formação de recursos humanos, nesse caso, para a promoção do DI saudável, valor 5 .

O presente estudo foi aprovado pelo Comitê de Ética em Pesquisa da Escola de Enfermagem da Universidade de São Paulo (CEP-EE-USP) (Processo nº 949/2010).

\section{RESULTADOS}

A caracterização do esquema analítico proposto foi organizada por cursos idênticos das IES partícipes, exceto Educação Física e Terapia Ocupacional. Nos gráficos a seguir, ressalta-se que as IC que não apresentam barras horizontais abordam o DI a partir de outros referenciais teóricos e metodológicos que, a priori, não promovem o cuidado integral e integrado.

O curso de Educação Física do CUC (Gráfico 1) apresentou as seguintes disciplinas: Teoria do lazer e recreação I, Teoria do lazer e recreação II, Crescimento e desenvolvimento e Aprendizagem motora, que abordam as especificidades da PI dos espaços de lazer e de interação com as famílias.

Gráfico 1 - Perfil da abordagem dos conteúdos da promoção do desenvolvimento infantil no curso de Educação Física antes dos projetos de intervenção local nos municípios do interior paulista. São Paulo, 2014.

EDUCAÇÃO FÍSICA

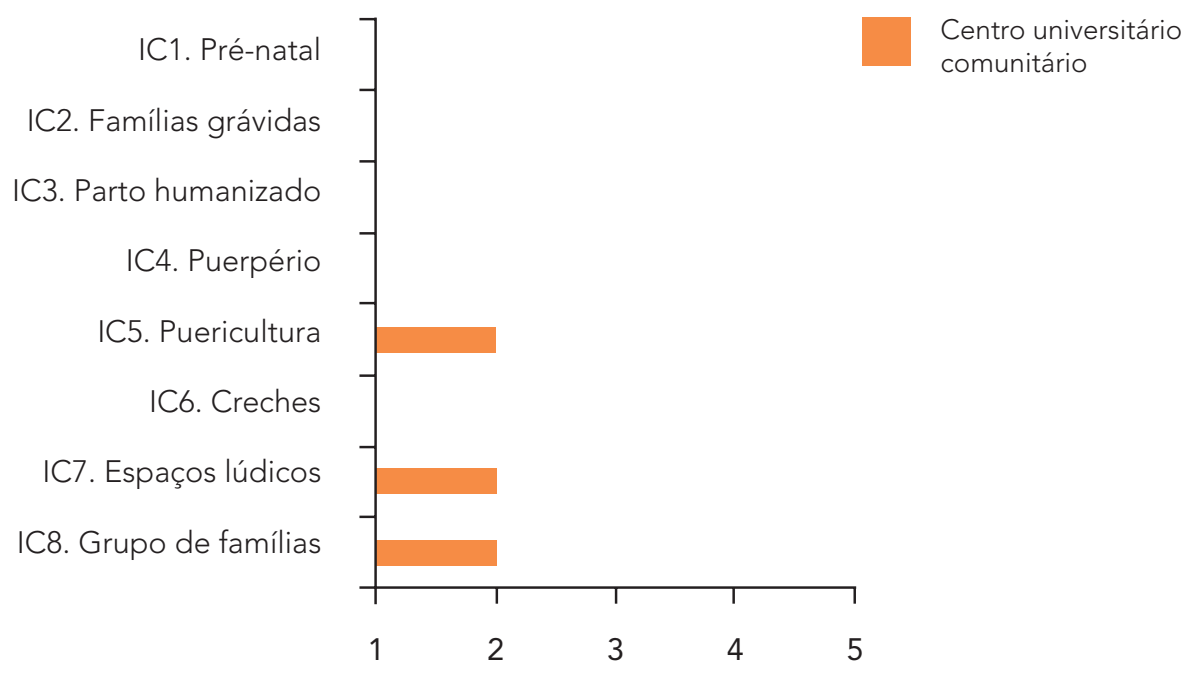


As disciplinas de Enfermagem da CUC são: Enfermagem no cuidado à criança, Enfermagem no cuidado à mulher e Enfermagem no cuidado ao recém-nascido. A abordagem do DI na Enfermagem da UFL ocorre em: Psicologia do desenvolvimento no ciclo vital, Propedêutica e processo de cuidar na saúde da criança e adolescente, Políticas de atenção à saúde da mulher, da criança e do adolescente, Enfermagem da família, Prática clínica do processo de cuidar na saúde da mulher, da criança e do adolescente.

Os dois cursos de Enfermagem analisados (Gráfico 2) têm o conteúdo relacionado ao ensino do DI em sete temáticas das IC, porém não abordam práticas de cuidado em creches. Contudo, produzem pesquisa em espaços na comunidade e na saúde da criança e da mulher.

Gráfico 2 - Perfil da abordagem dos conteúdos da promoção do DI nos cursos de Enfermagem antes dos PIL nos municípios do interior paulista. São Paulo, 2014.

\section{ENFERMAGEM}

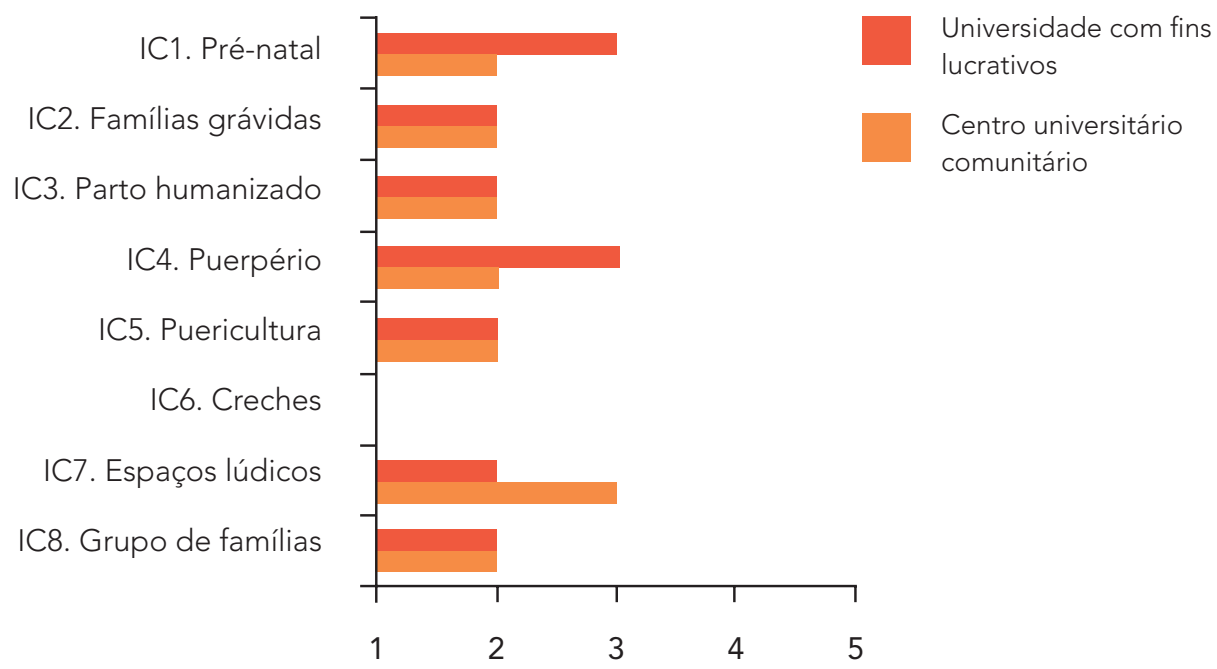

Os dados analisados (Gráfico 3 ) das disciplinas dos cursos de Fisioterapia da CUC (Psicologia geral do desenvolvimento e Fisioterapia aplicada à ginecologia, obstetrícia e pediatria), da UPF (Fisioterapia em ginecologia-obstetrícia e Fisioterapia em pediatria) e da UFL (Fisioterapia neurológica pediátrica e Psicologia aplicada à fisioterapia) apresentam maior variedade de abordagens do ensino do DI e convergem para o trabalho educativo reflexivo com pais e cuidadores. 
Gráfico 3 - Perfil da abordagem dos conteúdos da promoção do desenvolvimento infantil nos cursos de Fisioterapia antes dos projetos de intervenção local nos municípios do interior paulista. São Paulo, 2014.

\section{FISIOTERAPIA}

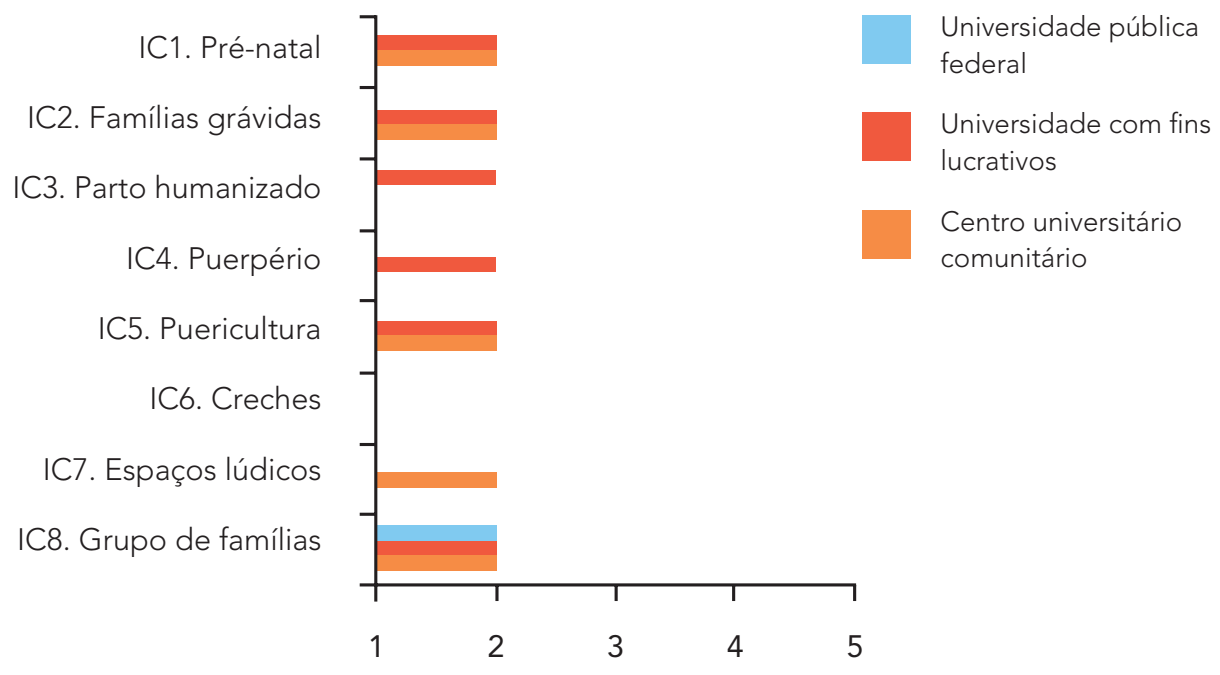

As disciplinas de Pedagogia analisadas (Gráfico 4) foram: Psicologia da educação, Oficina pedagógica/Educação infantil e Filosofia da educação I (CUC), Psicologia da aprendizagem, Estágio supervisionado II, Educação lúdica e Educação infantil (CUP), Psicologia do desenvolvimento e aprendizagem, Conteúdos, metodologias e práticas da educação infantil (FEP) e Prática na primeira infância [o-3 anos], Estrutura e organização da escola de educação infantil e Práticas e projetos na educação infantil [o-5 anos] (UFL).

Todas demonstram homogeneidade no ensino de práticas lúdicas nas comunidades e grupos interativos com pais e cuidadores e limitam-se ao ensino de quatro temáticas das intervenções-chave, não abordando conteúdos relacionados à saúde da criança. 
Gráfico 4 - Perfil da abordagem dos conteúdos da promoção do desenvolvimento infantil nos cursos de Pedagogia antes dos projetos de intervenção local nos municípios do interior paulista. São Paulo, 2014.

\section{PEDAGOGIA}

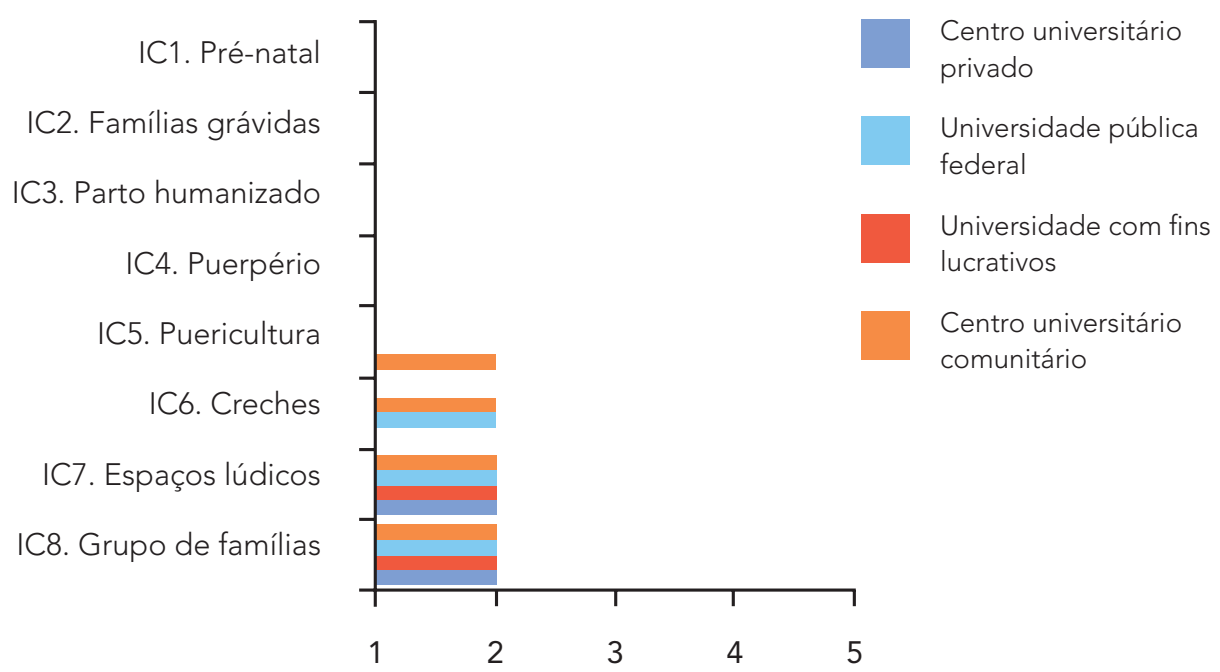

Os cursos de Psicologia analisados (Gráfico 5) enfatizam o ensino de sete temáticas das intervenções-chave e não abordam o cuidado no parto e puerpério, nas disciplinas a saber: Psicologia do desenvolvimento, Distúrbios psicológicos na infância e Psicologia familiar e conjugal (CUC); Psicologia da saúde, Psicologia e pessoas com necessidades especiais, Processos psicológicos básicos II, Neurociência básica e Psicologia do desenvolvimento na infância (CUP); Psicologia do desenvolvimento I, Psicologia do desenvolvimento II, Psicologia do desenvolvimento III, Psicologia e saúde e História social da família (FEP); e Psicologia do desenvolvimento: ciclo vital (UFL). 
Gráfico 5 - Perfil da abordagem dos conteúdos da promoção do desenvolvimento infantil nos cursos de Psicologia antes dos projetos de intervenção local nos municípios do interior paulista. São Paulo, 2014.

\section{PSICOLOGIA}

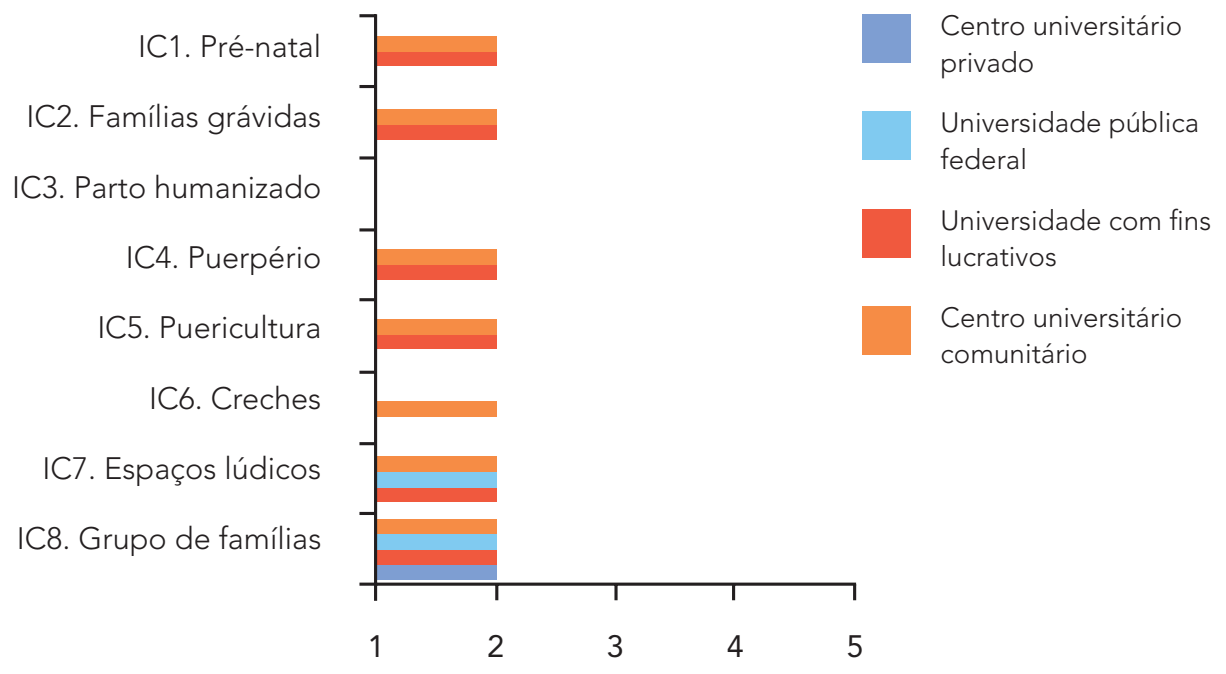

O curso de Terapia Ocupacional foi o único a apresentar currículo integrado e propiciar ações de extensão com foco em espaços comunitários para brincar e em grupos familiares (Gráfico 6). Desse modo, o corpo docente e os supervisores de estágio proporcionam aprendizado interativo e dinâmico no ensino de todos os conteúdos inovadores do DI por meio de Unidade de educação em prática supervisionada em TO I, Unidade de recursos teóricos e metodológicos em TO II e Unidade de recursos e atividades em TO II. 
Gráfico 6 - Perfil da abordagem dos conteúdos da promoção do desenvolvimento infantil no curso de Terapia Ocupacional antes dos projetos de intervenção local nos municípios do interior paulista. São Paulo, 2014.

\section{TERAPIA OCUPACIONAL}

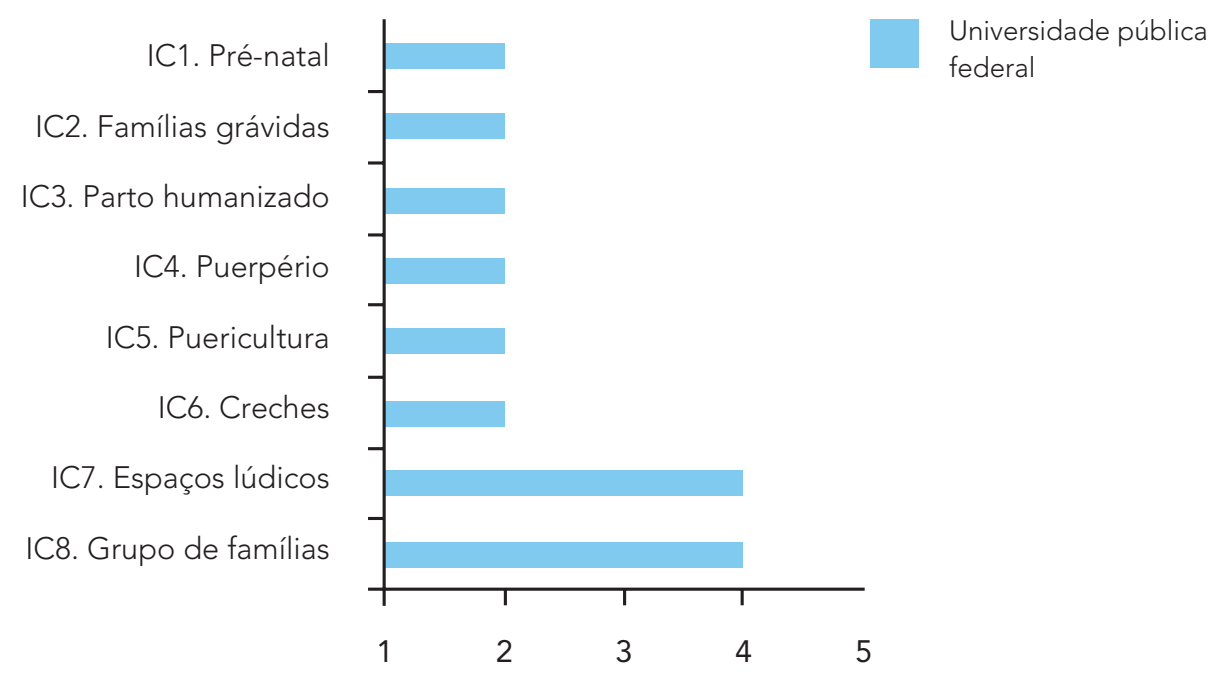

Observou-se a predominância do ensino do DI sem interfaces com a pesquisa e a extensão. Os cursos de Pedagogia não apresentaram conteúdos relacionados à promoção da saúde durante a PI, uma vez que os cuidados realizados na educação infantil devem extrapolar as noções de higiene e de cunho biológico e incorporar aspectos emocionais e socioambientais abordados nas $\mathrm{IC}_{1}$ a IC 4 .

\section{DISCUSSÃO}

A composição de um esquema analítico para avaliar a incorporação de tecnologias de cuidado inovadoras [5], que se fundamentam na síntese das evidências científicas relacionadas ao desenvolvimento infantil $[4,7,10,12,18,20]$, prima pela indissociabilidade como "um princípio orientador da qualidade da produção universitária, porque afirma como necessária a tridimensionalidade do fazer universitário autônomo, competente e ético" [15].

Diante do precedente, integrar elementos qualificáveis e quantificáveis que compõem a avaliação da extensão, que reconhece a interface com o ensino e a pesquisa, torna-se necessário para a proposição de formas inovadoras de sistematizar esse processo. A compreensão de Arroyo e Rocha [1] corrobora com as premissas do presente estudo, pois afirmam que: 
Os processos de avaliação reflexiva e crítica assumem um papel muito importante, porque podem/devem configurar-se como uma forma de saber com mais precisão o que se faz numa extensão, quem faz, como faz, para quem e por que faz. Além disso, a avaliação da extensão universitária não pode ser tratada de maneira isolada nas ações das IES, não só pela determinação das políticas atuais para o ensino superior, mas, também, porque quando se tem como meta a indissociabilidade entre ensino, pesquisa e extensão, a avaliação destes três eixos precisa ser feita de forma articulada.

A parceria com os projetos de intervenção local da Fundação Maria Cecília Souto Vidigal e os diferentes stakeholders dos municípios compreende um esforço para compor um contexto de avaliação da indissociabilidade para além dos muros das instituições de ensino superior, visto que Pedrazzi e Yamamoto [16] recomendam que:

Além da natureza acadêmica, cumprir ao preceito da articulação entre ensino, pesquisa e extensão (com integração da atividade de extensão desenvolvida à formação técnica e cidadã do estudante e pela produção e difusão de novos conhecimentos, novas técnicas e novas metodologias); ter, sempre que possível, características de interdisciplinaridade, pela interação de modelos e conceitos complementares, de material analítico e de metodologia, com ações interprofissionais e interinstitucionais [e intersetoriais], apresentando consistência teórica e operacional que permitam a viabilização das atividades de extensão.

A difusão de inovações, a transferência de tecnologias e a incorporação do conhecimento produzido por núcleos acadêmicos devem ser integradas na avaliação da tríade acadêmica. Marandino [11], no âmbito da educação não formal e da divulgação da ciência para a promoção do desenvolvimento cultural da população, reitera que:

Ainda são tímidas as avaliações dessas iniciativas [de extensão] e, em geral, estas não estão previstas nos financiamentos oferecidos pelas agências de fomento. Vemos aqui, novamente, o papel fundamental da universidade com potencial para desenvolver mecanismos qualitativos e quantitativos de análise e avaliação dessas ações, sendo esta mais uma forma de articulação entre pesquisa, ensino e extensão.

O crescente reconhecimento das potencialidades e dos benefícios decorrentes de parcerias entre academia e comunidade representa um avanço para as reflexões sobre a formação de profissionais competentes e cidadãos. Contudo, a exiguidade de processos avaliativos dessa interação acadêmica e comunitária demanda novas formas de sistematizar o conhecimento que pode se transformar em boas práticas profissionais, em políticas públicas e em ações que contribuam para o fortalecimento da confiança e da participação comunitária [2].

Dalben e Vianna [8], em ensaio teórico sobre a gestão institucional no ensino superior, recomendam que para a elaboração de avaliação das ações extensionistas "é necessário que a área da extensão construa indicadores auditáveis (possíveis de verificação), mensuráveis (valores numéricos) e comparáveis (indicadores únicos ou similares para todas as IES), de modo a conferir legitimidade ao processo".

O presente estudo reforça a indicação da utilização da abordagem qualitativa por 
múltiplos métodos para a avaliação e a construção de indicadores da parceria entre instituições de ensino superior (IES) e comunidade $[2,19]$ e pode ser descrito com os critérios acima [8], na forma de classificação para a (auto)avaliação de seus representantes (docentes, coordenadores, gestores, discentes e técnicos) comprometidos com a extensão bem articulada com o ensino e a pesquisa.

\section{CONCLUSÕES}

Levantar o perfil dos conteúdos relacionados ao desenvolvimento infantil (DI) nos cursos de graduação das IES partícipes proporcionou uma reflexão sobre a importância da assimilação das evidências científicas e da incorporação de novos conhecimentos nos diferentes cenários de cuidar, educar e brincar das crianças de zero a três anos a partir de uma parceria intersetorial que valoriza as potencialidades dos serviços locais, das famílias e das comunidades em prol da primeira infância (PI).

Essa parceria que envolveu diferentes stakeholders e, em especial, os estudantes de graduação e de pós-graduação, docentes, coordenadores e diretores das instituições de ensino superior (IES) partícipes, representa uma oportunidade com potencialidade para a formação de recursos humanos baseada em competências que articulam os serviços locais desde a graduação, sendo que a criação de oportunidades para o consumo do conhecimento (por exemplo, a síntese das evidências científicas mediadas pelas oito intervenções-chave promovidas pela Fundação Maria Cecília Souto Vidigal) pode representar uma forma de superação dessa lacuna entre as ações de extensão e de pesquisa na área da promoção do DI.

A ampliação do entendimento da PI como condição favorável ao desenvolvimento social deve extrapolar a centralidade do DI (em especial, nas IC 1 a 5 ) e agregar a produção de evidências científicas das diferentes áreas do conhecimento. Ao adotar tal concepção ampliada, as IES podem contribuir para a formação de força de trabalho através da tríade acadêmica que prima pelo fazer com as crianças e não apenas fazer sobre ou para elas.

As intervenções-chave 6 a 8 primam pela compreensão da criança como sujeito de direitos, plenas em sua inteireza e capazes de múltiplas formas de comunicação. Entretanto, essa dimensão da subjetividade na PI aparece pouco ou de forma incipiente na maioria dos cursos analisados. Torna-se essencial a discussão e a incorporação desses conteúdos inovadores na formação de futuros profissionais que efetivem práticas ampliadas para a PI.

Com base na classificação dos cursos analisados, a maior parte das IES ensina uma variedade dos conteúdos inovadores para a promoção do DI. Contudo, tornou-se evidente a ausência de incentivos para a integração de pesquisa e de extensão relacionada a esses conteúdos nos cenários avaliados, uma vez que apenas um dos cursos da universidade pública realizava atividades de extensão com foco na PI.

A produção do conhecimento pela pesquisa configura prática valorizada em determinados contextos acadêmicos. Nesse sentido, reconhece-se que as diferenças entre as naturezas administrativas das IES repercutem na indissociabilidade do ensino, 
pesquisa e extensão. Sugere-se realizar avaliação similar em núcleos acadêmicos confessionais, filantrópicos e públicos nas esferas estaduais e municipais a fim de ampliar a compreensão desse processo.

Torna-se imprescindível a construção de formas de sistematizar a incorporação de conteúdos, tecnologias e abordagens para fortalecer o cuidado infantil por meio das ações de extensão, sem desconsiderar o ensino e a pesquisa. Nessa direção, o esquema analítico apresentado pretende subsidiar novas formas de avaliar a sinergia dessas três dimensões das diferentes IES avaliadas e que pode ser verificado em outras áreas do conhecimento.

\section{REFERÊNCIAS}

[1] ARROYO, D. M. P.; DA ROCHA, M. S. P. M. L. Meta-avaliação de uma extensão universitária: Estudo de caso. Avaliação (Campinas), v.15, n.2, 2010. Disponível em: <http://www.scielo.br/scielo.php?script=sci_arttext\&pid $=$ S1414-40772010000200008 $>$. Acesso em 14 out. 2014. DOI: http://dx.doi. org/10.1590/S1414-40772010000200008

[2] BOWEN, S.; MARTENS, P. J. A model for collaborative evaluation of university-community partnerships. J Epidemiol Community Health, v. 6o, p. 902-907, 2006. Disponível em: <http://www.ncbi.nlm.nih.gov/pmc/articles/ PMC2566062/>. Acesso em 14 out. 2014. DOI: 10.1136/jech.2005.040881

[3] BRASIL. Fórum de Pró-Reitores de Extensão das Universidades Públicas Brasileiras. Avaliação Nacional da Extensão Universitária. Brasília, 2001.

[4] CENTER ON THE DEVELOPING CHILD, NATIONAL SCIENTIFIC COUNCIL ON THE DEVELOPING CHILD, NATIONAL FORUM ON EARLY CHILDHOOD POLICY AND PROGRAMS. The Foundations of Lifelong Health Are Built in Early Childhood. Harvard: Center on the Developing Child, [s.d.].

[5] CHIESA, A.M. et al. A construção de tecnologias de atenção em saúde com base na promoção da saúde. Rev. Esc. Enferm. USP [online], v. 43, n. 2, p. 13521357, 2009. Disponível em: <http://www.scielo.br/scielo.php?script=sci_artte xt\&pid=Soo8o-62342009000600036>. Acesso em 14 out. 2014. DOI: http:// dx.doi.org/10.1590/Soo8o-62342009000600036

[6] CORRÊA, E.J. (Org.). Fórum de Pró-Reitores de Extensão das Universidades Públicas Brasileiras, Coordenação Nacional do FORPROEX. Extensão Universitária: organização e sistematização. Belo Horizonte: Coopmed, 2007.

[7] CYPEL, S. (Org.). Fundamentos do desenvolvimento infantil: da gestação aos 3 anos. São Paulo: Fundação Maria Cecília Souto Vidigal, 2011.

[8] DALBEN, A. I. L. F.; VIANNA, P. C. M. Gestão e avaliação da extensão universitária: a construção de indicadores de qualidade. Interagir: pensando a extensão, v. 13, p. 31-39, 2008. Disponível em: <http://www.e-publicacoes.uerj. br/index.php/interagir/article/view/1669/1312>. Acesso em 14 out. 2014.

[9] FRENK, J. et al. Health professionals for a new century: transforming education 
to strengthen health systems in an interdependent world. The Lancet, v. 376, n. 9756, p. 1923-1958, 2010. Disponível em: <http://www.thelancet.com/journals/ lancet/article/PIISo140-6736(10)61854-5/fulltext?_eventId=login >. Acesso em 14 out. 2014. DOI: 10.1016/So140-6736(10)61854-5

[10] FUNDAÇÃO MARIA CECÍLIA SOUTO VIDIGAL. Projetos de Intervenção Local: Estratégias para qualificar a atenção à Primeira Infância. São Paulo, 2011.

[11] MARANDINO, M. Educação, Ciência e Extensão: A Necessária Promoção. Rev. Cult. Ext. USP, n. 9, p. 89-100, 2013. Disponível em: <file://C:/Users/ auditorio-03/Downloads/56642-71514-1-SM.pdf >. Acesso em 14 out. 2014. DOI: http://dx.doi.org/10.11606/issn.2316-906o.v9iop89-10o

[12] MARMOT, M.; ATKINSON, T.; BELL, J. Fair Society, healthy lives: The Marmot Review. Strategic review of health inequalities in England Post, 2010.

[13] MEYER, J. Usando métodos qualitativos na pesquisa-ação relacionada à saúde. In: POPE, C.; MAYS, N. (Org.). Pesquisa qualitativa na atenção à saúde. 2. ed. Porto Alegre: Artmed, 2005. p. 71-86.

[14] MINAYO, M. C. S. O desafio do conhecimento: pesquisa qualitativa em saúde. 8.ed. São Paulo: Hucitec, 2004.

[15] MOITA, F. M. G. S. C.; ANDRADE, F. C. B. Ensino-pesquisa-extensão: um exercício de indissociabilidade na pós-graduação. Rev. Bras. Educ, v.14, n.41, p. 269-280, 2009. Disponível em: <http://www.scielo.br/scielo.php?pid=S1413$-24782009000200006 \&$ script $=$ sci_abstract\&tlng=pt $>$. Acesso em 14 out. 2014. DOI: http://dx.doi.org/10.1590/S1413-24782009000200006

[16] PEDRAZZI, V.; YAMAMOTO, M. M. A necessidade de ações articuladas na Cultura e Extensão. Rev. Cult. Ext. USP, n. 10, p. 43-50, 2013. Disponível em: <file:///C:/Users/auditorio-03/Downloads/69049-91062-2-PB.pdf>. Acesso em 14 out. 2014. DOI: 10.11606/issn.2316-9060.v10iop43-50

[17] SANTOS, B.S. A universidade no século XXI: para uma reforma democrática e emancipatória da universidade. 2.ed. São Paulo: Cortez, 2005.

[18] SHONKOFF, J. P. et al. The lifelong effects of early childhood adversity and toxic stress. Pediatrics, n. 129, p. 232-246, 2012. Disponível em: <http://pediatrics.aappublications.org/content/129/1/e232.full.pdf >. Acesso em 14 out. 2014. DOI: $10.1542 /$ peds.2011-2663

[19] THIOLENT, M. A inserção da pesquisa-ação no contexto da extensão universitária. In: BRANDÃO, C.R.; STRECK, D.R. (Orgs.). Pesquisa participante: a partilha do saber. Aparecida: Ideias e Letras, 2006. p.151-165.

[20] YOUNG, M.E. (Org.). Do desenvolvimento da primeira infância ao desenvolvimento humano: investindo no futuro de nossas crianças. São Paulo: Fundação Maria Cecília Souto Vidigal, 2010. 
AMC atua como consultora técnica do Programa de Desenvolvimento Infantil da Fundação Maria Cecília Souto Vidigal. Os demais autores declaram a participação em atividades dos projetos de intervenção local promovidos pela FMCSV.

\section{AGRADECIMENTOS}

Os autores agradecem à Fundação de Amparo à Pesquisa do Estado de São Paulo pelo financiamento para a realização da pesquisa intitulada $A$ universidade e a transferência de tecnologias de desenvolvimento infantil em municípios paulistas (processo FAPESP no 2010/09263-6) e à Fundação Maria Cecília Souto Vidigal pela oportunidade de criar e fortalecer ações nos municípios do interior paulista em prol do desenvolvimento saudável na primeira infância.

ALFRED ALMEIDA PINA-OLIVEIRA enfermeiro, doutorando e mestre em Saúde Coletiva da Escola de Enfermagem da Universidade de São Paulo (EE-USP) - e-mail: alfredopina@usp.br

ANNA MARIA CHIESA enfermeira e professora associada do Departamento de Enfermagem em Saúde Coletiva da Escola de Enfermagem da Universidade de São Paulo (EE-USP) - e-mail: amchiesa@usp.br

ROSELI DE LANA MOREIRA enfermeira e mestranda em Saúde Coletiva da Escola de Enfermagem da Universidade de São Paulo (EE-USP) - e-mail: roseli.lana@hotmail.com

ROSEMARY APARECIDA FRACOLLI PÉCORA enfermeira e mestre em Ciências da Saúde da Escola de Enfermagem da Universidade de São Paulo (EE-USP) - e-mail: rosemary_fracolli@ yahoo.com.br 